\title{
X-Ray Holographic Imaging of Hydrated Biological Cells in Solution
}

\author{
M. Bartels, M. Krenkel, J. Haber, R. N. Wilke, and T. Salditt \\ Institut für Röntgenphysik, Georg-August-Universität Göttingen, Friedrich-Hund-Platz, 1, Göttingen 37077, Germany
}

(Received 3 August 2014; revised manuscript received 14 November 2014; published 28 January 2015)

\begin{abstract}
We demonstrate nanoscale $\mathrm{x}$-ray holographic imaging using optimized illumination wave fronts emitted by $\mathrm{X}$-ray waveguide channels. Mode filtering minimizes wave-front distortions and artifacts encountered in most hard x-ray focusing schemes, enabling quantitative reconstruction of the projected density, as evidenced by a test pattern imaged with a field of view of about $20 \times 40 \mu \mathrm{m}$ and at $22 \mathrm{~nm}$ resolution. The dose efficiency and contrast sensitivity make the optical scheme compatible with samples of intrinsically low contrast, typical for hydrated soft matter. This is demonstrated by imaging bacteria in the hydrated and living state, with quantitative phase contrast revealing dense structures of the bacterial nucleoids associated with compactified DNA. In response to continued irradiation, characteristic changes in these dense structures are observed.
\end{abstract}

DOI: 10.1103/PhysRevLett.114.048103

PACS numbers: 87.59.-e, 42.40.-i, 87.14.gk, 87.53.-j

Imaging of biological matter at the nanoscale is characterized by three persistent challenges: resolution, contrast, and compatibility with functional or physiological conditions. For the investigation of biological cells-which are often referred to as the test tubes of the 21 st centuryimaging of processes and functions is particularly essential. Hard x-ray coherent imaging [1-7] is unique as a probe of the native electron density distribution within cells and thicker tissue. It is compatible with a large range of environmental conditions, does not depend on labeling or staining, and is well suited for tomography of larger specimens, due to a high penetration power and a large depth of focus. After overcoming considerable challenges related to the phase problem (Refs. [6,7] and the references therein), the resolution achievable with lensless coherent $\mathrm{x}$-ray diffractive imaging (CDI) has become high enough to address subcellular architectures [8-14], such as the topology of biological membranes in complex organelles, the organization of protein networks, and compactified DNA.

A major challenge of applying $\mathrm{x}$-ray imaging to biological matter is the low contrast in the hydrated state and the high radiation dose's inducement of radiation damage. Most reported dose values, even for dehydrated cells with strong contrast, are in the range of $10^{7}-10^{9} \mathrm{~Gy}$, well above the theoretical dose-resolution curve which increases with a power law of exponent $3 \leq \gamma \leq 4$, as derived for the case of Fraunhofer far-field diffraction [15]. Such excessive dose values are prohibitive for cells in solution, let alone for live cell imaging. A recent soft x-ray CDI study has demonstrated imaging of mammalian cells under low dose

Published by the American Physical Society under the terms of the Creative Commons Attribution 3.0 License. Further distribution of this work must maintain attribution to the author(s) and the published article's title, journal citation, and DOI. conditions [16], but it was limited to the freeze-dried state. A first CDI study of cells in solution ("wet" CDI) reported $30 \mathrm{~nm}$ resolution (stated as half-period throughout this Letter), but at a "cost" of $10^{8}$ Gy [17].

In this Letter we present a different approach to nanoscale x-ray imaging, at a drastically reduced dose and with a large field of views, based on in-line holographic recordings using optimized and filtered wave fronts. The method is demonstrated here using first a lithographic test pattern imaged at a resolution of $22 \mathrm{~nm}$, and second the grampositive bacteria Deinococcus radiodurans in the freezedried state, at a resolution of $53 \mathrm{~nm}$ and a radiation dose of $10^{4} \mathrm{~Gy}$. Coherent $\mathrm{x}$-ray imaging provides a unique tool to shed light on the disputed structural arrangement of DNA in the nucleoid of this bacterium $[18,19]$. From the quantitative density contrast, constraints on DNA packing models can be obtained [13], complementing electron microscopy studies [20]. The dense, round structures observed by x-ray imaging within freeze-dried Deinococcus radiodurans cells $[13,14,21]$ were attributed to DNA rich regions in the bacterial nucleoids. Here, we show that these structures can be imaged even in the hydrated and living state. To this end, we present the first electron density maps of living cells in buffered solution that were obtained by an application of less than the lethal dose. Successive images reveal structural processes in the nucleoids in response to radiation. This result casts serious doubt on previous conclusions that radiation damage does not change the observed structure of hydrated cells on the $50 \mathrm{~nm}$ scale for the typical high dose values of CDI [17] and underlines the need for dose-efficient imaging approaches.

To achieve phase contrast images at a drastically reduced dose and nanoscale resolution, we use $\mathrm{x}$-ray full-field imaging with contrast formation by free space propagation in combination with highly coherent and well-controlled 
spherical wave fronts emitted by $\mathrm{x}$-ray waveguides [22,23]; see the sketch in Fig. 1. Introduced almost two decades ago [1,24], $\mathrm{x}$-ray propagation imaging uses phase reconstruction algorithms $[6,7,25,26]$ to invert the intensity pattern(s) recorded downstream from an object illuminated by a plane wave, or spherical wave, for the sake of geometric magnification [27-29]. However, the wave-front errors associated with hard $\mathrm{x}$-ray focusing lead to a severe loss of image quality and, to correct for this, we here use waveguide mode filtering, which significantly reduces wave-front aberrations and increases the spatial coherence. Progress in fabrication of lithographic waveguide channels has enabled us to overcome the previously low efficiency of $\mathrm{x}$-ray waveguide optics [30], increasing the waveguide exit flux of the present experiment to $I_{\mathrm{WG}}>10^{9} \mathrm{ph} / \mathrm{sec}$. Since the sample is not positioned in a focus but at a defocus position, the flux density at the sample can be adjusted to a tolerable level. The optical setup is combined with optimized near-field phase retrieval algorithms to achieve quantitative reconstructions from a single hologram.

Dose-efficient imaging of weakly diffracting objects becomes possible due to two distinct features of waveguide holography: (i) The homogeneous signal level within the recorded radiation cone circumvents well-known challenges associated with the limited dynamical range of x-ray detectors and does not require the use of beamstops; (ii) the waveguide transmits only the radiation modes required for the coherent imaging process and filters out background radiation, which is absorbed in the cladding [31]. The waveguide thus protects the sample from unwanted incoherent radiation which would not improve image quality but would increase the dose. Furthermore, interference of the weak diffracted wave behind the sample with the much stronger and highly coherent primary wave enhances the signal level well above the background originating at or downstream from the sample, including the Compton background of the sample itself [32]. In addition, the magnified near-field (Fresnel) diffraction pattern (in-line hologram) directly represents the location and the shape of the object, enabling easy sample alignment and providing a further optional constraint for iterative phase retrieval.

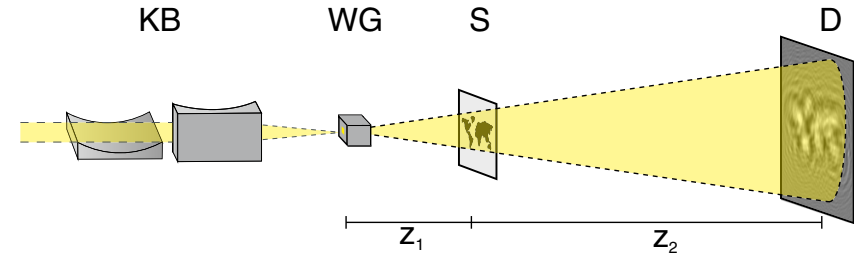

FIG. 1 (color online). A monochromatic hard x-ray beam is focused by Kirkpatrick-Baez (KB) mirrors onto a waveguide (WG) system. The sample (S) is illuminated by the waveguide beam and magnified Fresnel diffraction patterns are recorded at the detection plane (D).
The experiments were performed using the GINIX instrument [33] at the coherence beam line $P 10$ of the PETRA III storage ring (Hamburg, Germany; see Ref. [34] for details). The undulator beam was monochromatized [Si(111)] and focused by Kirkpatrick-Baez (KB) mirrors to about $300 \mathrm{~nm}$ in the horizontal and vertical directions. The twodimensional $x$-ray waveguides were placed in the focal plane of the mirrors, acting as a spatial and coherence filter [31]. The samples were placed into the divergent wave field exiting the waveguide, at a distance $z_{1}$. In-line holograms, magnified by a factor of $M=1+z_{2} / z_{1}$, were recorded using a fiber coupled sCMOS detector (Photonic Science) with a pixel size of $P=6.54 \mu \mathrm{m}$, positioned in the detector plane at $z_{1}+z_{2} \approx 5 \mathrm{~m}$ behind the waveguide. This is equivalent to a parallel beam case with an effective sample-detector distance $z=z_{2} / M$ and a demagnified pixel size $p=P / M$. The recorded holographic intensity $I_{z}(x, y):=\left|\mathcal{D}_{z}\{P(x, y) \cdot O(x, y)\}\right|^{2}$ can be calculated based on the free space Fresnel propagator $\mathcal{D}_{z}$ acting on the product between object transmission function $O$ and probe function $P$, which in turn emerges from the waveguide exit field. Figure 2(a) shows a scanning electron micrograph of the exit surface of a 1-mm-long bonded silicon waveguide. The waveguide exit flux of $2 \times 10^{9}$ photons/s was determined from the measured far-field pattern shown in Fig. 2(b). The source size (FWHM) of

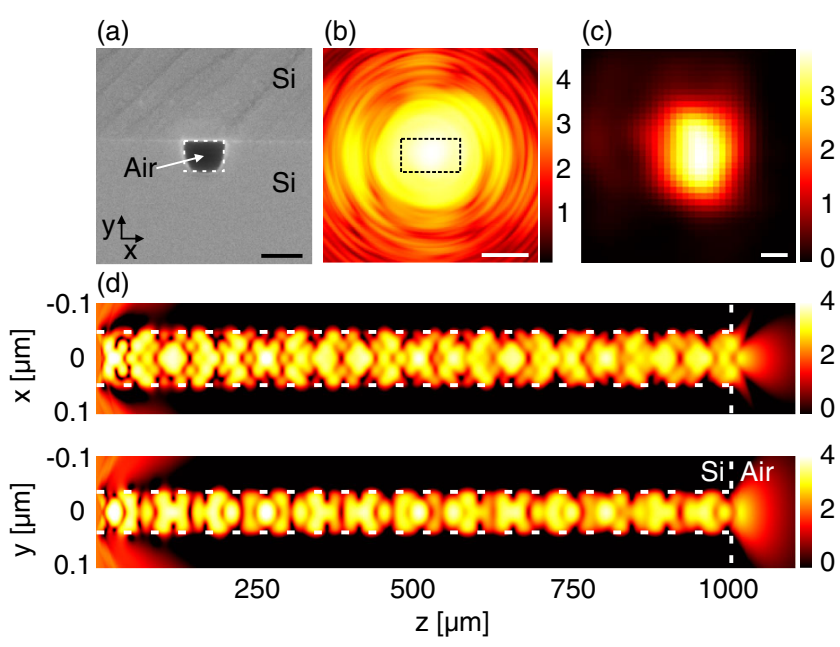

FIG. 2 (color online). Silicon channel waveguide fabricated by electron-beam lithography, followed by wafer bonding. (a) Scanning electron micrograph of the exit surface. The channel cross section is enclosed by the dashed rectangle $(97 \times 73 \mathrm{~nm})$. (b) Logarithmic far-field intensity distribution, as measured in photon numbers by a pixel detector about $5 \mathrm{~m}$ behind the waveguide. The smooth central part (the dashed rectangle) is used for imaging. (c) Reconstructed near-field intensity, linear color coding. (d) Simulated intensity distribution along the beam direction $(z)$ within and right behind a two-dimensional waveguide with channel dimensions $d_{x}=97 \mathrm{~nm}$ and $d_{y}=73 \mathrm{~nm}$, logarithmic color coding. Scale bars (a) $100 \mathrm{~nm}$, (b) $10 \mathrm{~mm}$, (c) $20 \mathrm{~nm}$. 
$25.4 \mathrm{~nm}$ (horizontal) $\times 30.8 \mathrm{~nm}$ (vertical) was determined from the near-field reconstruction shown in Fig. 2(c), using the error-reduction algorithm [23]. The small source sizecompared to the channel dimensions of $d_{x}=97 \mathrm{~nm}$ and $d_{y}=73 \mathrm{~nm}$-arises based on multimodal interference, as supported by the finite difference simulations shown in Fig. 2(d). Full details on the bonded silicon waveguide (the air channel) used for holographic recordings at $7.9 \mathrm{keV}$ and the $\mathrm{Ge} / \mathrm{Mo} / \mathrm{C} / \mathrm{Mo} / \mathrm{Ge}$ waveguide system used for the $13.8 \mathrm{keV}$ recordings are given in Ref. [34].

The waveguide exit beam is fully coherent $[31,33]$ and has a smooth Gaussian-like line shape [Figs. 2(b) and 2(c)]. Propagation of the empty waveguide beam is therefore well approximated by a pure geometrical enlargement (considering amplitudes), and image formation can be expressed as $I_{z}:=\left|\mathcal{D}_{z}\{P \cdot O\}\right|^{2} \simeq\left|\mathcal{D}_{z}\{P\}\right|^{2} \cdot\left|\mathcal{D}_{z}\{O\}\right|^{2}$. This enables artifact-free normalization by the empty beam $I_{z}^{E}:=\left|\mathcal{D}_{z}\{P\}\right|^{2}$, expressed by $\bar{I}_{z}=I_{z} / I_{z}^{E}=\left|\mathcal{D}_{z}\{O\}\right|^{2}$. The normalized intensity is thus directly related to the object transmission function $O=\exp \left[-i 2 \pi / \lambda \int_{-\Delta t}^{0}\left[\delta_{\lambda}(x, y, z)-\right.\right.$ $\left.\left.i \beta_{\lambda}(x, y, z)\right] d z\right]$ of the object with thickness $\Delta t$ and refractive index $n=1-\delta_{\lambda}+i \beta_{\lambda}$ at wavelength $\lambda$, in contrast, for example, to cone-beam holography experiments with $\mathrm{KB}$ beams [28], where the empty beam intensity normalization fails [36].

To benchmark the optical setup and phase retrieval algorithms, we have first imaged a test pattern milled by focusing an ion beam into a 200-nm-thick gold layer on a 200-nm-thick $\mathrm{Si}_{3} \mathrm{~N}_{4}$ membrane. Figure 3(a) shows the normalized hologram $\bar{I}_{z}$ of a 200 second exposure recorded at $13.6 \mathrm{keV}$ photon energy, sample distance $z_{1}=4.93 \mathrm{~mm}$ and detector distance $z_{1}+z_{2}=5.07 \mathrm{~m}$. Interference fringes extend all the way to the corners of the diffraction pattern, indicating a high quality hologram. The different reconstructions of the object phase $\varphi(x, y)$ are shown in Figs. 3(b), 3(c), and 3(d). The holographic reconstruction (a)

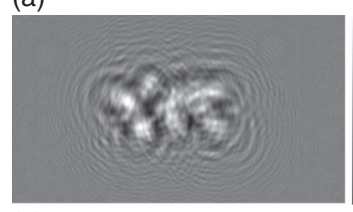

(c)

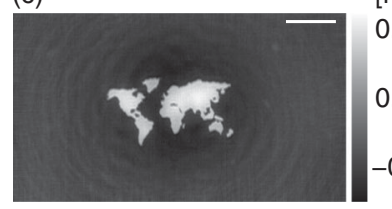

(b)

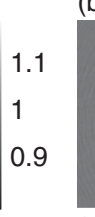

[rad] (d)

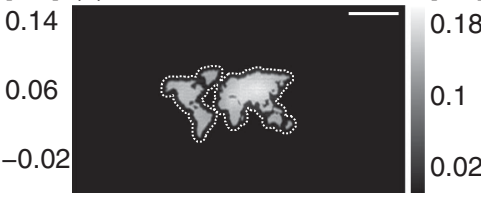

FIG. 3. (a) Normalized hologram of a test structure milled into $200 \mathrm{~nm}$ thick gold. (b) Holographic phase reconstruction. (c) Phase reconstruction based on the contrast transfer functions (CTF). (d) Iterative mHIO phase reconstruction, the support information (the dashed line) was obtained from the reconstruction shown in (c). Scale bars, $2 \mu \mathrm{m}$. $\varphi(x, y)=\varphi\left\{\mathcal{D}_{-z}\left\{\bar{I}_{z}\right\}\right\}$, based on the free space Fresnel diffraction operator $\mathcal{D}_{z}$, is shown in Fig. 3(b) and exhibits the well-known twin-image artifacts of in-line holography. For objects with a slowly varying phase and negligible absorption, the image formation can be linearized and written in Fourier space as $\tilde{I}_{z}\left(\nu_{x}, \nu_{y}\right) \simeq \delta_{\mathrm{D}}\left(\nu_{x}, \nu_{y}\right)+$ $2 \tilde{\varphi}\left(\nu_{x}, \nu_{y}\right) \sin [\chi(\nu, z)]$, where $\tilde{a}=\mathcal{F}\{a\}$ denotes the two-dimensional Fourier transform, $\nu_{x}, \nu_{y}$ the spatial frequencies with $\nu^{2}=\nu_{x}^{2}+\nu_{y}^{2}$, and $\delta_{D}$ the Dirac delta function. The term $\sin [\chi(\nu, z)]$ with $\chi(\nu, z)=\pi \lambda z \nu^{2}$ is known as the phase contrast transfer function (CTF). Phase reconstruction via filtering in Fourier space based on the CTF [24] suppresses the twin-image artifacts, as shown in Fig. 3(c). However, because of zeros in the phase $\mathrm{CTF}$ at $\nu_{0}=\sqrt{n / \lambda z}$, with $n \in \mathbb{N}$, some pronounced artifacts remain, in particular at low spatial frequencies. Here we use the CTF reconstruction to initialize a modified hybrid input-output (mHIO) algorithm which is capable of recovering the missing information [26], based on the support of the object, which is readily inferred from the deterministic CTF reconstruction. In essence, the algorithm propagates back and forth between sample and detection plane, using a numerical implementation of the free space Fresnel diffraction operator $\mathcal{D}_{z}$ with $\psi_{z}=\mathcal{D}_{z}\left\{\psi_{0}\right\}=$ $\mathcal{F}^{-1}\left\{\exp (\mathrm{i} 2 \pi z / \lambda) \exp \left(-\mathrm{i} \pi \lambda z \nu^{2}\right) \mathcal{F}\left\{\psi_{0}\right\}\right\}$, and enforces compact object support as well as intensity values in line with the measured data, respectively. As shown in Fig. 3(d), the phase distribution after $N_{\text {it }}=1000$ iterations reveals the object nearly artifact free. The world map exhibits sharp edges and uniform gray values. A $100 \times 100$ pixel domain yields a mean phase shift of $\mu_{\varphi}=0.18 \mathrm{rad}$ and a standard deviation $\sigma_{\varphi}$, with $\sigma_{\varphi} / \mu_{\varphi}=3 \%$. In addition to the absence of low frequency artifacts, the superior quality of the mHIO reconstruction also manifests itself in an increased resolution of $22 \mathrm{~nm}$ (compared to $24 \mathrm{~nm}$ for the CTF reconstruction), as determined by fits to edges at different regions of the object. See Ref. [34] for additional information on data processing and reconstruction algorithms.

After optimization of experimental settings and algorithms, the approach was used to image freeze-dried bacteria. Cells of the Deinococcus radiodurans strain $R 1$ were cultivated from freeze-dried cultures and vitrified on $\mathrm{Si}_{3} \mathrm{~N}_{4}$ foils by plunge freezing them in liquid ethane, followed by freeze drying as in Ref. [14], as detailed in Ref. [34]. Samples were imaged at $7.9 \mathrm{keV}$, using the smooth central cone of the waveguide field shown in Fig. 2, well matched to the active area of the sCMOS detector [the dashed rectangle in Fig. 2(b)], placed at $z_{1}+z_{2}=5.12 \mathrm{~m}$. A single 8 second accumulation of the sample placed at $z_{1}=15.9 \mathrm{~mm}$ was recorded, along with a corresponding empty beam measurement. Figure 4(a) shows the normalized hologram of a group of Deinococcus radiodurans cells (without any further data treatment), while Fig. 4(b) depicts 
(a)

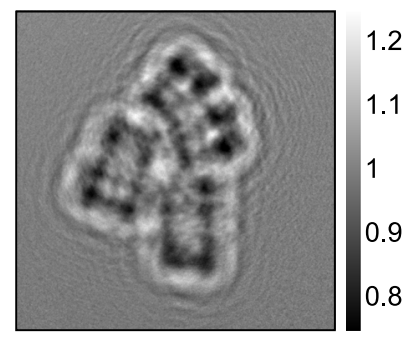

(c) $-0.08=0$ [rad]

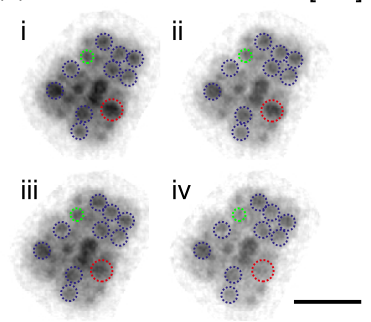

(b)

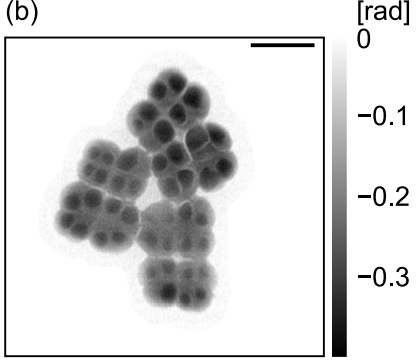

(d)

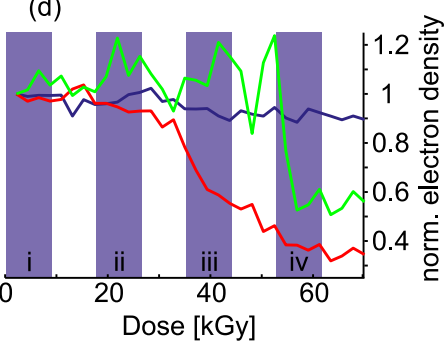

FIG. 4 (color online). (a) Normalized hologram of freeze-dried Deinococcus radiodurans cells, obtained in a single recording with $8 \mathrm{~s}$ dwell time along with (b) the iterative $\mathrm{mHIO}$ phase reconstruction. (c) mHIO reconstruction of (initially) living cells in solution. Each frame was accumulated for $8 \times 10$ seconds (every other frame is shown). Pronounced changes in the densities are observed after successive irradiation, as quantified in (d), showing the normalized electron density in the high density nucleoid regions indicated by the corresponding colors as a function of dose. The images in (c) are reconstructions corresponding to averages over the colored columns in (d). Scale bars, $4 \mu \mathrm{m}$.

a mHIO reconstruction after $N_{\text {it }}=741$ iterations. The diskshaped domains with large relative phase shifts of up to -0.3 radian can be clearly identified and attributed to the bacterial nucleoid. With an effective pixel size of $20.3 \mathrm{~nm}$, the crossover to the noise plateau of the power spectral density (PSD) at about 0.19 cycles per pixel corresponds to a resolution of about $53 \mathrm{~nm}$; see Ref. [34]. The flux density at the sample plane was $5 \times 10^{5} \mathrm{ph} / \mu \mathrm{m}^{2} / \mathrm{s}$, corresponding to a total dose of $D=5.2 \times 10^{3}$ Gy applied during 8 seconds, as calculated for model protein [15]. This is almost 3 orders of magnitude less than a recent ptychographic reconstruction of the same bacteria (of the same preparation batch) at similar photon energy $(6.2 \mathrm{keV})$, contrast, and resolution $(50 \mathrm{~nm})$, recorded at a dose of $4.9 \times$ $10^{6}$ Gy [14]. And, in contrast to ptychographic scanning [14,37], a large field of view, e.g., of $(20 \mu \mathrm{m})^{2}$, is observed simultaneously, which is important for samples in semistable environments or dynamic states, e.g., hydrated or living samples.

Since the freeze-dried cells were imaged below the lethal dose of Deinococcus radiodurans, the next step was to image living bacteria in solution. For the measurement, the bacteria were kept in microscopy chambers compatible with cell culture (ibidi, Germany); see Ref. [34] for details.

At a photon energy of $13.8 \mathrm{keV}, 56$ images with 10 second exposure time were recorded with the sample placed at $z_{1}=19.7 \mathrm{~mm}$, corresponding to an effective pixel size of $25.4 \mathrm{~nm}$. Eight consecutive exposures were averaged, yielding 7 frames with 80 second accumulation time for each. Figure 4(c) shows the reconstruction for every other frame of the live cell recordings. To increase the signal-tonoise ratio, the holograms were binned by a factor of 2 . Phase reconstruction of each frame was performed using the mHIO algorithm with 3500 iterations, on average. Resolution is degraded due to slight sample movement in the solution during the exposure and is estimated to about 2 to 3 pixels, corresponding to $100-150 \mathrm{~nm}$. The results confirm that the dense round structures attributed to the nucleoids observed in the freeze-dried state [see Fig. 4(c) and Refs. [13,14,21]] are also present in the hydrated living state of the bacterium. With a total flux of $2 \times 10^{7} \mathrm{ph} / \mu \mathrm{m}^{2}$ in each frame, the dose is $D=8.9 \times 10^{3}$, as calculated for a model protein [15], and $D=8.6 \times 10^{3}$ for water. These values are below the lethal dose $L D_{50}>10^{4}$ of Deinococcus radiodurans. The retrieved electron density map of at least the first frame should, therefore, represent the native structure in the living state of the bacteria, while in successive frames, radiation induced changes in the density distribution can be monitored. Notably, the density of the nucleoids decreases, but quite differently for individual organelles, as quantified in Figs. 4(c) and 4(d). While most nucleoids are subject to gradual density fading, for some organelles the process occurs in pronounced steps; see the colored columns in Fig. 4(d).

Importantly, the applied dose could be precisely adjusted and reduced without a breakdown of the phase retrieval process. Despite the dose reduction by orders of magnitude with respect to most far-field diffractive imaging studies reported previously, including studies of the same organism $[11,13,14]$, we could already observe radiation induced structural changes in the course of consecutive exposures. In contrast to previous claims of wet CDI [17], we conclude that imaging of living or hydrated biological samples at $50 \mathrm{~nm}$ resolution is not possible, in general, without severe damage. At the same time, the onset of radiation induced processes and subsequent radiation damage could be precisely studied with the demonstrated dose-efficient holographic approach. This may enable future studies of repair processes in response to radiation damage, from a structural point of view. The role of possible cofactors could be investigated, e.g., by varying the buffer solution or the metabolic state of the bacteria. For single low dose exposures, the method enables the visualization of the subcellular density distribution within living cells, even in complex environments. This structural probe could then be enhanced by well-chosen nanodiffraction spots, yielding high resolution in reciprocal space $[14,38]$. Last but not least, and beyond the single cell level, this dose-efficient holographic approach should also enable 3D reconstruction 
with subcellular resolution in tissues, based on nested phase and tomographic reconstruction, for example [39].

We thank Michael Sprung for the support at the $P 10$ beamline, and the German Research Foundation (DFG) for funding through Grant No. SFB 755/C1.

*tsaldit@gwdg.de

[1] S. W. Wilkins, T. E. Gureyev, D. Gao, A. Pogany, and A. W. Stevenson, Nature (London) 384, 335 (1996).

[2] J. Miao, P. Charalambous, J. Kirz, and D. Sayre, Nature (London) 400, 342 (1999).

[3] F. Pfeiffer, T. Weitkamp, O. Bunk, and C. David, Nat. Phys. 2, 258 (2006).

[4] P. Thibault, M. Dierolf, A. Menzel, O. Bunk, C. David, and F. Pfeiffer, Science 321, 379 (2008).

[5] C. Holzner, M. Feser, B. Hornberger, S. B. Baines, and C. Jacobsen, Nat. Phys. 6, 883 (2010).

[6] D. Paganin, Coherent X-Ray Optics (Oxford University Press, Oxford, 2006).

[7] K. A. Nugent, Adv. Phys. 59, 1 (2010).

[8] D. Shapiro et al., Proc. Natl. Acad. Sci. U.S.A. 102, 15343 (2005).

[9] C. Song, H. Jiang, A. Mancuso, B. Amirbekian, L. Peng, R. Sun, S. S. Shah, Z. Hong Zhou, T. Ishikawa, and J. Miao, Phys. Rev. Lett. 101, 158101 (2008).

[10] Y. Nishino, Y. Takahashi, N. Imamoto, T. Ishikawa, and K. Maeshima, Phys. Rev. Lett. 102, 018101 (2009).

[11] E. Lima, L. Wiegart, P. Pernot, M. Howells, J. Timmins, F. Zontone, and A. Madsen, Phys. Rev. Lett. 103, 198102 (2009).

[12] X. Huang et al., Phys. Rev. Lett. 103, 198101 (2009).

[13] K. Giewekemeyer, P. Thibault, S. Kalbfleisch, A. Beerlink, C. M. Kewish, M. Dierolf, F. Pfeiffer, and T. Salditt, Proc. Natl. Acad. Sci. U.S.A. 107, 529 (2010).

[14] R. N. Wilke, M. Priebe, M. Bartels, K. Giewekemeyer, A. Diaz, P. Karvinen, and T. Salditt, Opt. Express 20, 19232 (2012).

[15] M. R. Howells et al., J. Electron Spectrosc. Relat. Phenom. 170, 4 (2009).

[16] M. W. M. Jones et al., Ultramicroscopy 143, 88 (2014).

[17] D. Nam et al., Phys. Rev. Lett. 110, 098103 (2013).

[18] E. Eltsov and J. J. Dubochet, J. Bacteriol. 188, 6052 (2006).

[19] A. Minsky, E. Shimoni, and J. Englander, J. Bacteriol. 188, 6059 (2006).
[20] S. Levin-Zaidman, J. Englander, E. Shimoni, A. K. Sharma, K. W. Minton, and A. Minsky, Science 299, 254 (2003).

[21] M. Bartels, M. Priebe, R. N. Wilke, S. P. Krüger, K. Giewekemeyer, S. Kalbfleisch, C. Olendrowitz, M. Sprung, and T. Salditt, Opt. Nanosc. 1, 10 (2012).

[22] S. P. Krueger, K. Giewekemeyer, S. Kalbfleisch, M. Bartels, H. Neubauer, and T. Salditt, Opt. Express 18, 13492 (2010).

[23] S. P. Krueger, H. Neubauer, M. Bartels, S. Kalbfleisch, K. Giewekemeyer, P. J. Wilbrandt, M. Sprung, and T. Salditt, J. Synchrotron Radiat. 19, 227 (2012).

[24] P. Cloetens, W. Ludwig, J. Baruchel, D. Van Landuyt, J. P. Guigay, and M. Schlenker, Appl. Phys. Lett. 75, 2912 (1999).

[25] T. E. Gureyev, Opt. Commun. 220, 49 (2003).

[26] K. Giewekemeyer, S. P. Krueger, S. Kalbfleisch, M. Bartels, C. Beta, and T. Salditt, Phys. Rev. A 83, 023804 (2011).

[27] M. Bartels, V. H. Hernandez, M. Krenkel, T. Moser, and T. Salditt, Appl. Phys. Lett. 103, 083703 (2013).

[28] R. Mokso, P. Cloetens, E. Maire, W. Ludwig, and J.-Y. Buffière, Appl. Phys. Lett. 90, 144104 (2007).

[29] M. W. M. Jones et al., Sci. Rep. 3, 2288 (2013).

[30] A. Jarre, C. Fuhse, C. Ollinger, J. Seeger, R. Tucoulou, and T. Salditt, Phys. Rev. Lett. 94, 074801 (2005).

[31] M. Osterhof and T. Salditt, New J. Phys. 13, 103026 (2011).

[32] J. M. Slowik and R. Santra, J. Phys. B 46, 164016 (2013).

[33] T. Salditt, S. Kalbfleisch, M. Osterhoff, S. P. Krueger, M. Bartels, K. Giewekemeyer, H. Neubauer, and M. Sprung, Opt. Express 19, 9656 (2011).

[34] See Supplemental Material at http://link.aps.org/ supplemental/10.1103/PhysRevLett.114.048103, which includes Refs. [31,35], for more detailed information on methods and procedures.

[35] C. Fuhse and T. Salditt, Appl. Opt. 45, 4603 (2006).

[36] J. Hagemann, A.-L. Robisch, D. R. Luke, C. Homann, T. Hohage, P. Cloetens, H. Suhonen, and T. Salditt, Opt. Express 22, 11552 (2014).

[37] M. Dierolf, A. Menzel, P. Thibault, P. Schneider, C. M. Kewish, R. Wepf, O. Bunk, and F. Pfeiffer, Nature (London) 467, 436 (2010).

[38] B. Weinhausen, O. Saldanha, R. N. Wilke, C. Dammann, M. Priebe, M. Burghammer, M. Sprung, and S. Köster, Phys. Rev. Lett. 112, 088102 (2014).

[39] A. Ruhlandt, M. Krenkel, M. Bartels, and T. Salditt, Phys. Rev. A 89, 033847 (2014). 